\title{
A note on absorption semigroups and regularity
}

\author{
Amir Manavi*, Hendrik Vogt ${ }^{\dagger}$ and Jürgen Voigt ${ }^{\ddagger}$
}

\begin{abstract}
It is shown that, in the theory of absorption semigroups, two possible ways of defining regularity for absorption rates are in fact equivalent.
\end{abstract}

MSC2010: 47D06, 47A55, 47B60

Keywords: Absorption semigroup, regular absorption rate

\section{Introduction}

The notion of 'absorption semigroup' was introduced in [6] in the context of absorption rates for positive $C_{0}$-semigroups acting on $L_{p}$-spaces. Let $(\Omega, \mu)$ be a measure space. Given a positive $C_{0}$-semigroup $T=(T(t))_{t \geqslant 0}$ on $L_{p}(\mu)$ with generator $A$, the aim was to associate a $C_{0}$-semigroup with the formal expression $A-V$ as generator, for measurable $V: \Omega \rightarrow \mathbb{R}$ as general as possible.

One of the properties turning out to be of importance was the regularity of absorption rates $V: \Omega \rightarrow[0, \infty)$. On the one hand, it was used to compute the generator of the perturbed semigroup in the case $p=1$ (cf. [6; Corollary 4.3(b)]), and it was also used to show form properties of Schrödinger operators (cf. [6; Proposition 5.8(b)]). On the other hand, in [7; Theorem 3.5] a seemingly stronger version of regularity was used to show a dominated convergence theorem for absorption semigroups.

It is the purpose of the present note to show that the two versions of regularity are in fact equivalent.

We mention that the concepts sketched above have been treated in more generality in [3], for absorption semigroups on Banach function lattices, and in [4; Chapter 2], for the case of propagators instead of semigroups.

\footnotetext{
*Goethestr. 7, 71332 Waiblingen, Germany, a.manavi@gmx.de

†Fachbereich Mathematik, Universität Bremen, Postfach 330 440, 28359 Bremen, Germany, hendrik.vogt@uni-bremen.de

$\ddagger$ Technische Universität Dresden, Fachrichtung Mathematik, 01062 Dresden, Germany, juergen.voigt@tu-dresden.de
} 


\section{Equivalence of two notions of regularity}

Let $(\Omega, \mathcal{A}, \mu)$ be a measure space, let $p \in[1, \infty)$, and let $T$ be a positive $C_{0^{-}}$ semigroup on $L_{p}(\mu)$, with generator $A$. The following notions have been introduced in $[6$.

If $V: \Omega \rightarrow[0, \infty]$ or $V: \Omega \rightarrow[-\infty, 0]$, then $V$ is called $T$-admissible if $V$ is locally measurable,

$$
T_{V}(t):=\mathrm{s}_{n \rightarrow \infty} \lim ^{t(A-(V \wedge n) \vee(-n))}
$$

exists for all $t \geqslant 0$, and $T_{V}$ thus defined constitutes a $C_{0}$-semigroup. We note that then the convergence stated in (1.1) is uniform for $t$ in bounded subsets of $[0, \infty)$, in short expressed as $T_{V}=\mathrm{s}-\lim _{n \rightarrow \infty} T_{(V \wedge n) \vee(-n)}$. The generator of $T_{V}$ will be denoted by $A_{V}$.

For an absorption rate $V: \Omega \rightarrow[0, \infty]$ we recall two notions of regularity: $V$ is called $T$-regular if $V$ is $T$-admissible and

$$
T_{0, V}:=\underset{\varepsilon \rightarrow 0+}{\operatorname{s}-\lim } T_{\varepsilon V}=T .
$$

$V$ is called strongly $T$-regular if $V$ is $T$-admissible and

$$
\left(T_{V}\right)_{-V}=\underset{n \rightarrow \infty}{\mathrm{s}-\lim _{V-V \wedge n}} T_{V}
$$

(The first equality in the previous line is always valid; this follows from [6; Lemma 2.4].) Regularity was defined in [6; Definition 2.12]. Strong regularity was defined in [7; Definition 3.1] (under the notion 'regular'); we adopt the terminology 'strongly regular' from [2; Definition 2.3]. The following result shows that the above two notions are equivalent.

1.1 Theorem. (cf. [3; Satz 4.1.54]) Let $V: \Omega \rightarrow[0, \infty]$ be T-admissible. Then

$$
T_{0, V}=\left(T_{V}\right)_{-V}
$$

In particular, $V$ is $T$-regular if and only if $V$ is strongly $T$-regular.

Proof. Let $0<\varepsilon \leqslant 1$. Then $\varepsilon V=\varepsilon(V \wedge n)+\varepsilon(V-V \wedge n) \leqslant \varepsilon n+V-V \wedge n$ for all $n \in \mathbb{N}$, and this implies $T_{\varepsilon V} \geqslant T_{\varepsilon n+V-V \wedge n}=\mathrm{e}^{-\varepsilon n \cdot} T_{V-V \wedge n}$; recall [6; Remark 2.1(a)]. Taking first $\varepsilon \rightarrow 0$ and then $n \rightarrow \infty$, we obtain $T_{0, V} \geqslant\left(T_{V}\right)_{-V}$.

In order to show the reverse inequality we note that $T \geqslant\left(T_{V}\right)_{-V} \geqslant T_{V}$ implies strong continuity of $\left(T_{V}\right)_{-V}$; so $-V$ is $T_{V}$-admissible. From 7; Proposition 3.3(b)] we therefore conclude that $V$ is strongly $T_{V}$-regular. Then [7; Proposition 3.3(a)] implies that all positive multiples of $V$ are strongly $T_{V}$-regular. The application of this fact with $\varepsilon V$ instead of $V$ shows that $V$ is strongly $T_{\varepsilon V}$-regular for all $\varepsilon>0$. From [7; Proposition 1.3(a)] it follows that

$$
\left(T_{\varepsilon V}\right)_{V-V \wedge n} \leqslant T_{V-V \wedge n}
$$


and taking $n \rightarrow \infty$ in this inequality we infer $T_{\varepsilon V} \leqslant \mathrm{~s}-\lim _{n \rightarrow \infty} T_{V-V \wedge n}=\left(T_{V}\right)_{-V}$, for all $\varepsilon>0$. Letting $\varepsilon \rightarrow 0$ we obtain $T_{0, V} \leqslant\left(T_{V}\right)_{-V}$.

The last assertion of the theorem is a consequence of the previous equality and the very definitions of regularity and strong regularity.

1.2 Remark. In [6] and [7, the notions of admissibility and regularity have only been considered for absorption rates $V$ taking their values in $\mathbb{R}$. It was observed in [1] that it is more appropriate to allow for extended real-valued absorption rates $V$. It was shown in [1; Proposition 4.4] that for a $T$-admissible absorption rate $V: \Omega \rightarrow[0, \infty]$ the set $[V=\infty]$ is a local null set. Also, it can be shown as in [7. Proposition 3.3(b)] that for a $T$-admissible absorption rate $V: \Omega \rightarrow[-\infty, 0]$ the absorption rate $-V$ is $T$-admissible, and therefore $[V=-\infty]$ is a local null set.

In view of these observations, for an extended positive or negative $T$-admissible absorption rate $V: \Omega \rightarrow[-\infty, \infty]$ one can always replace $V$ by an equivalent absorption rate taking its values in $\mathbb{R}$, and therefore the results of [6], [7] are applicable.

We conclude the paper by showing that - in a certain sense - the hypothesis of $T$-admissibility in the definition of regularity is redundant; see Theorem 1.3 and Remark 1.4 below. In order to make this precise we have to extend the definition of $T_{V}$ to arbitrary locally measurable $V: \Omega \rightarrow[0, \infty]$. Indeed, the limit

$$
T_{V}(t):=\underset{n \rightarrow \infty}{\operatorname{sim}} \mathrm{e}^{t(A-V \wedge n)}
$$

exists for all $t \geqslant 0$ (recall [6; Remark 2.1(c)]), and $T_{V}$ thus defined is a oneparameter semigroup. It was shown in [1; Corollary 3.3] that then $P:=$ $\mathrm{s}-\lim _{t \rightarrow 0+} T_{V}(t)$ exists and is a band projection. This implies that there exists a decomposition $\mu=\mu_{1}+\mu_{2}$ with $\mu_{1} \perp \mu_{2}$ (' $\perp$ ' meaning 'locally disjoint') such that $R(P)=L_{p}\left(\mu_{1}\right)$. (We refer to [5] for this description of projection bands in $L_{p}(\mu)$.) It follows that the restriction of $T_{V}$ to $L_{p}\left(\mu_{1}\right)$ exists and is a $C_{0}$-semigroup.

By $\left(T_{V}\right)_{-V}$ we denote the $C_{0}$-semigroup obtained from $\left.T_{V}\right|_{L_{p}\left(\mu_{1}\right)}$ by applying $-V$ as a perturbation. (Note that $-V$ is $\left.T_{V}\right|_{L_{p}\left(\mu_{1}\right)}$-admissible because $T_{V \wedge m-V \wedge n} \leqslant T$ for all $m \geqslant n \geqslant 0$.) We also extend $\left(T_{V}\right)_{-V}(t)$ to $L_{p}(\mu)$ as $J\left(T_{V}\right)_{-V}(t) P$, for $t>0$, where $J$ denotes the injection of the band $R(P)=L_{p}\left(\mu_{1}\right)$ into $L_{p}(\mu)$.

1.3 Theorem. Let $V: \Omega \rightarrow[0, \infty]$ be locally measurable, and assume that $\left(T_{V}\right)_{-V}(t)=T(t)$ for all $t>0$.

Then $V$ is $T$-admissible.

Proof. The hypothesis implies $P=\mathrm{s}-\lim _{t \rightarrow 0+}\left(T_{V}\right)_{-V}(t)=\mathrm{s}-\lim _{t \rightarrow 0+} T(t)=I$. Therefore $T_{V}$ is a $C_{0}$-semigroup on $L_{p}(\mu)$. 
1.4 Remark. Assuming solely that $V: \Omega \rightarrow[0, \infty]$ is locally measurable, one can show pretty much as in the proof of Theorem 1.1 that $\left(T_{V}\right)_{-V}(t)=\mathrm{s}_{-} \lim _{\varepsilon \rightarrow 0+} T_{\varepsilon V}(t)$ for all $t>0$. We mention that in the course of this proof one also obtains $\mathrm{s}-\lim _{t \rightarrow 0+} T_{\varepsilon V}(t)=P$ for all $\varepsilon \in(0,1]$.

\section{References}

[1] W. Arendt and C.J.K. Batty: Absorption semigroups and Dirichlet boundary conditions. Math. Ann. 295, 427-448 (1993).

[2] M. Ishikawa: Admissible and regular potentials for positive $C_{0}$-semigroups and application to heat semigroups. Semigroup Forum 48, 96-106 (1994).

[3] A. Manavi: Zur Störung von dominierten $C_{0}$-Halbgruppen auf Banachfunktionenräumen mit ordnungsstetiger Norm und sektoriellen Formen mit singulären komplexen Potentialen. Dissertation (doctoral thesis), TU Dresden, 2001.

[4] H. Vogt: Perturbation theory for parabolic equations. Habilitationsschrift, TU Dresden, 2010.

[5] H. Vogt and J. Voigt: Bands in $L_{p}$-spaces. In preparation.

[6] J. Voigt: Absorption semigroups, their generators, and Schrödinger semigroups. J. Funct. Anal. 67, 167-205 (1986).

[7] J. Voigt: Absorption semigroups. J. Operator Theory 20, 117-131 (1988). 